\title{
Classification Systems for Wave Energy Resources and WEC Technologies
}

Vincent S. Neary" ${ }^{\# 1}$ Ryan G. Coe ${ }^{\# 2}$, João Cruz ${ }^{* 3}$, Kevin Haas ${ }^{+4}$, Giorgio Bacelli ${ }^{\# 5}$, Yannick Debruyne ${ }^{* 6}$, Seongho $\mathrm{Ahn}^{+7}$, Victor Nevarez ${ }^{\# 8}$

${ }^{\#}$ Sandia National Laboratories

Albuquerque, NM, USA

1vsneary@sandia.gov

${ }^{2}$ rcoe@sandia.gov

${ }^{5}$ gbacell@sandia.gov

${ }^{8}$ vnevare@sandia.gov

${ }^{*}$ Cruz Atcheson Consulting Engineers, Lda.

Lisbon, Portugal

3joao.cruz@cruzatcheson.com

${ }^{6}$ yannick.debruyne@cruzatcheson.com

${ }^{+}$Georgia Institute of Technology

Atlanta, GA, USA

${ }^{4}$ khaas@gatech.edu

${ }^{7}$ seongho.ahn@gatech.edu

\begin{abstract}
Classification systems for wave energy resources and wave energy converter (WEC) technologies could provide similar benefits to those for the wind energy industry: resource classification facilitating reconnaissance studies and project planning at both regional and national scales; and WEC classification streamlining and reducing costs of WEC device design and manufacturing. In the present study, a classification system for U.S. wave resources is used to investigate the feasibility of WEC classification. Wave spectra inputs from three wave energy resource classes delineated in this system are used to derive distributions of optimized WEC design scaling factors, as well as WEC design responses. Preliminary results indicate that a single standard WEC design class could serve within a given resource class, and corresponding regional wave climate, due to distinct wave energy distributions and concentrations of energy within partitioned period bands for each resource class. The WEC response to extreme loads was found to vary considerably within the most energetic of the resource classes examined, suggesting the need for these standard design classes to meet structural design requirements based on the upper limits of load response within a given resource class. However, the observed load metric variation is lower than the inter-region resource variations.
\end{abstract}

Keywords - Wave Energy Converter (WEC); Wave Climate; Wave Resource Classification, WEC Classification; Site Conditions; Engineering Design.

\section{INTRODUCTION}

The success of wind energy resource and wind turbine classification systems has motivated interest in classification systems for the wave energy industry. A wave energy resource classification system would serve as a useful tool that facilitates scoping studies and project planning at both regional and national scales. A wave energy converter (WEC) classification scheme, ideally coupled with resource classification, would reduce design and manufacturing costs while minimizing technical and commercial risk. However, it is not entirely clear how to develop these wave energy classification systems in a way that provides similar benefits as those introduced in the wind energy industry. Like a wind turbine, a WEC should be designed to optimize power capture per unit cost, as well as maximize operational reliability and survive over a reasonable service life. However, unlike a wind turbine, the WEC's ability to maximize power capture through resonance with the incident waves may drive the size of the device. These conflicting requirements, coupled with the nature of the wave resource, introduce significant loading variations and creates a nontrivial design challenge.

Classification of three-bladed horizontal axis wind turbines appears to emphasize operational reliability and survival under extreme load conditions, with turbine classes delineated using a reference wind speed representing an extreme 10-minute average wind speed occurring every 50 -years on average, and subclasses determined by an expected turbulence intensity [1]. However, due to the high correlation between extreme wind speeds and mean wind speeds, this classification system also incorporates design considerations for average power generation. A preliminary WEC classification scheme recently presented by Cruz [2] delineated three wave energy resource classes (classes III to I, increasing in energy content) based on plots of mean sea state, i.e., significant wave height vs. mean peak period, for over three-thousand wave sites in the United Kingdom and Ireland. As mean significant wave height and wave energy density generally increase with mean peak period, the classes represent sites with increasing energy content from the lowest class (III), having the lowest mean peak period band, 
to the highest class (I) having the highest period band. Preliminary WEC classes, corresponding to these wave energy resource classes, were proposed for specific WEC archetype subclasses, e.g., two subclasses of a point absorber, based on the root-mean-squared power-take-off (PTO) force derived from the extreme $75^{\text {th }}$ percentile significant wave height for each wave energy resource class. As WEC classification was the main thrust of this study, metrics used for energy resource classification included only the mean significant wave height and mean peak period, which combined define a mean sea state from which other useful energy resource statistics, e.g., mean wave power density, and extreme (high-percentile) sea state statistics, can be derived.

The wave energy resource classification system, as presented herein, classifies the resource based on the amount of energy (or power) available within the dominant partitioned peak period band containing the largest energy fraction. It also classifies the wave energy resource with a relative risk metric, where risks (characterized by the extreme significant wave height with a 50-year recurrence interval) are normalized by the mean significant wave height. Maps of the period partitioned available energy and relative significant wave height reveal distinct regional trends.

The goal of this study is to investigate classification schemes for wave energy resources and WEC technologies. Initially a resource classification scheme is developed using key wave climate statistics, including those that characterize opportunities for wave power conversion, and those that characterize relative risks to WEC operation and survival. This resource classification provides the foundation for examining the potential feasibility of a WEC technology classification scheme, determined through assessment of the spread (variance) of both the optimized WEC design scaling factor, and WEC extreme load responses.

The potential feasibility of WEC design classification was assessed in three basic steps. First, the U.S. wave climate (resource) was classified using several metrics to characterize and discriminate unique wave climate conditions. WEC design optimization and design response tools were then used to derive distributions of design scaling factors and extreme load responses within each of these identified wave resource classes. The methodologies and key results are presented sequentially in the following sections.

\section{WAVE RESOURCE CLASSIFICATION}

Wave resource classification statistics were derived from validated phase II 30-year hindcast outputs using the 3rd generation (3G) spectral wave model, WaveWatch III® (WWIII), at a spatial resolution of 4 min [3]. Partitioned wave height, peak period and mean direction were outputted at every grid point. Individual wave heights and peak periods are calculated for each partition representing either the local wind sea or individual swell components.

Rather than classify a site's resource by significant wave height $\left(H_{s}\right)$ and energy period $\left(T_{e}\right)$, as was done by Cruz (2015), the present study uses the annual available energy (AAE) density, in $\mathrm{MWh} / \mathrm{m}$, as the main classification parameter. The
AAE density is the annual energy production (AEP) density assuming no energy conversion losses. It also accounts for the distribution of the AAE density within distinct partition period band classes. The significant wave height $\left(H_{s}\right)$ and energy period $\left(T_{e}\right)$ for each partition are used to calculate the wave power density $(J)$ of that partition,

$$
J=\frac{\rho g}{16} H_{s}^{2} C_{g}\left(T_{e}, h\right)
$$

where $C_{g}$ is the group velocity, a function of $T_{e}$ and the depth (h). The annual available energy (AAE) density is then calculated as a function of peak period $\left(T_{p}\right)$

$$
\operatorname{AAE}\left(\mathrm{T}_{\mathrm{p}}\right)=\mathrm{T}_{\text {year }} \sum J\left(T_{p}\right) f\left(J, T_{p}\right)
$$

where $\mathrm{T}_{\text {year }}$ is the number of hours in a year (8766 hours), and $f\left(J, T_{p}\right)$ is the joint probability of the partitioned wave power density and peak periodf $\left(J, T_{p}\right)$. The total AAE density is the summation over all peak periods

$$
\mathrm{AAE}=\sum \mathrm{AAE}\left(\mathrm{T}_{\mathrm{p}}\right)
$$

The mean annual wave power density, therefore, is defined as the total AAE density divided by the number of hours in a year. The total AAE and power densities are shown in Fig. 1. The largest values exceed $300 \mathrm{MWh} / \mathrm{m}$ along the West Coast, the south coast of Alaska, and Hawaii. AAE densities are generally between 50 and $200 \mathrm{MWh} / \mathrm{m}$ along the East Coast, and below $50 \mathrm{MWh} / \mathrm{m}$ along the Gulf Coast. The AAE density is used to define four broad resource classes shown in Fig. 2: Class 0 sites (AAE $<10 \mathrm{MWh} / \mathrm{m})$, representing sites having consistently low power that would only support very specialized energy projects, e.g., powering remote sensors, Class 1 sites $(10<\mathrm{AAE}<50$ $\mathrm{MWh} / \mathrm{m}$ ), with generally low power that might support specialized application, e.g., desalinization, and Class 2 $(50<\mathrm{AAE}<200 \mathrm{MWh} / \mathrm{m})$ and Class 3 (200<AAE MWh/m) sites with moderate to high power supporting utility scale projects.

The resource classification system herein distributes the AAE density within four different period bands. These period bands correspond to local wind seas (Band 1, $T_{p}<7 \mathrm{~s}$ ), short period swell (Band 2, $7 \mathrm{~s}<T_{p}<10 \mathrm{~s}$ ), moderate period swell (Band 3,10s $<T_{p}<14 \mathrm{~s}$ ), and long period swell (Band 4, $14 \mathrm{~s}<T_{p}$ ). This delineation of AAE density by period bandwidth allows WEC designers to distinguish sites where energy is concentrated within a dominant period bandwidth, a desirable resource attribute, as opposed to sites where the energy is spread relatively more evenly among multiple period bands.

The geographic distributions of AAE/power density among these period bands are shown in Fig. 3. As expected local wind seas (Band 1) contribute little AAE energy density in any U.S. wave climate, and are the main source of wave energy (approximately half or more) in the Gulf Coast. Short period swell contributes the main source of energy in the East Coast 
wave climate, and moderate period swell contributes the main source of energy for wave climates in the West Coast.

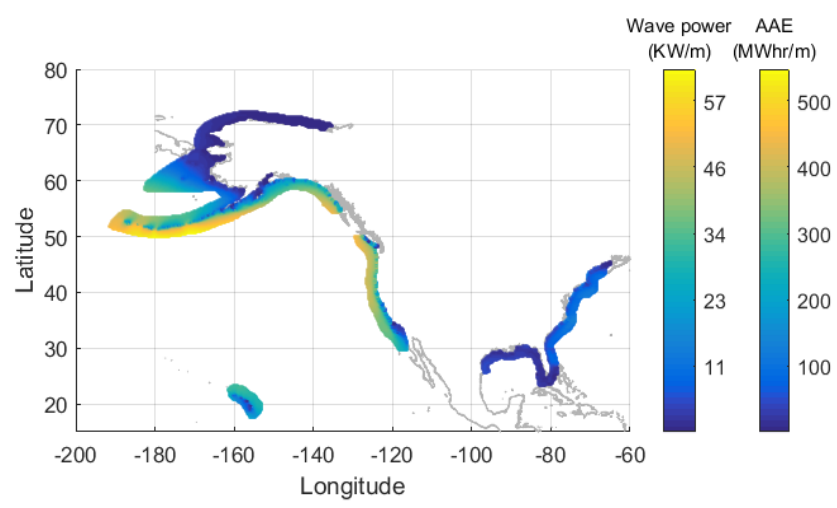

Fig. 1. Geographic distribution of total AAE Density and mean annual wave power density.

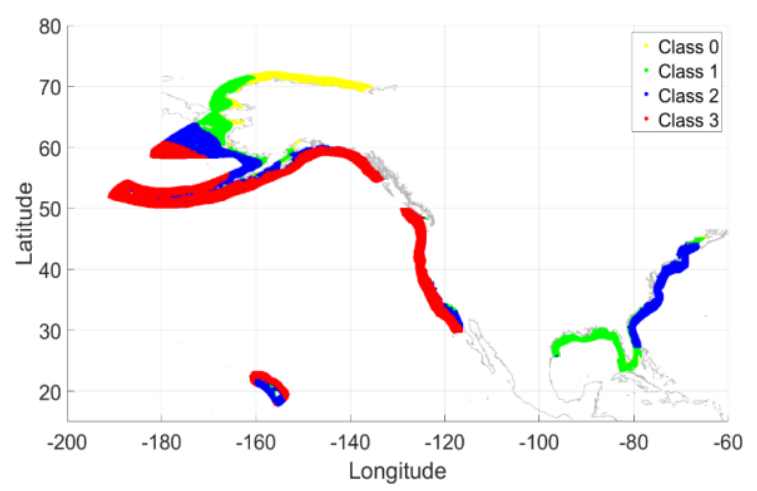

Fig. 2. Geographic distribution of wave energy resource classes.

The resource classification system also distinguishes three different relative risk classes based on the correlation between $H_{S_{50}}$ and $H_{s_{\text {mean }}}$, and the ratio $H_{s_{50}} / H_{s_{\text {mean }}}$, where $H_{S_{50}}$ is the significant wave height with a 50 -year return period and $H_{s_{\text {mean }}}$ is the mean significant wave height. The relative risk metric, $H_{S_{50}} / H_{s_{\text {mean }}}$, is analogous to the turbulence intensity used to delineate wind resource subclasses. Mapping this relative risk metric, $H_{s_{50}} / H_{s_{\text {mean }}}$, one can distinguish regional trends shown in Fig. 4. The West Coast, Hawaii, and most of the southern coast of Alaska, are predominantly low relative risk wave climates. The East Coast, west coast of Alaska and Western Gulf Coast are classified as medium relative risk climates, and the Eastern Gulf Coast and northern coast of Alaska are classified as high relative risk climates. Generally, the more energetic the wave climate, the lower the relative risk.

In summary, key resource attributes, including AAE/power density and the relative risk metric, $H_{S_{50}} / H_{s_{\text {mean }}}$, were calculated and mapped for all U.S. coastal waters. These maps indicate distinct regional wave climates, with distinct classes of resources. In the following analysis, wave spectra time series from thirty wave sites in three U.S. regional wave climates (ten sites for each climate) are used as inputs in WEC design optimization algorithms to derive distributions of WEC scaling factors, and to evaluate the feasibility of developing standard
WEC design classes. For this preliminary investigation, spectra from ten sites uniformly distributed within a regional wave climate were selected for three different wave climates to provide the broadest range of resource conditions: The West Coast, a predominantly Class 3 resource, the East Coast (Class 2), and Gulf Coast (Class 1).
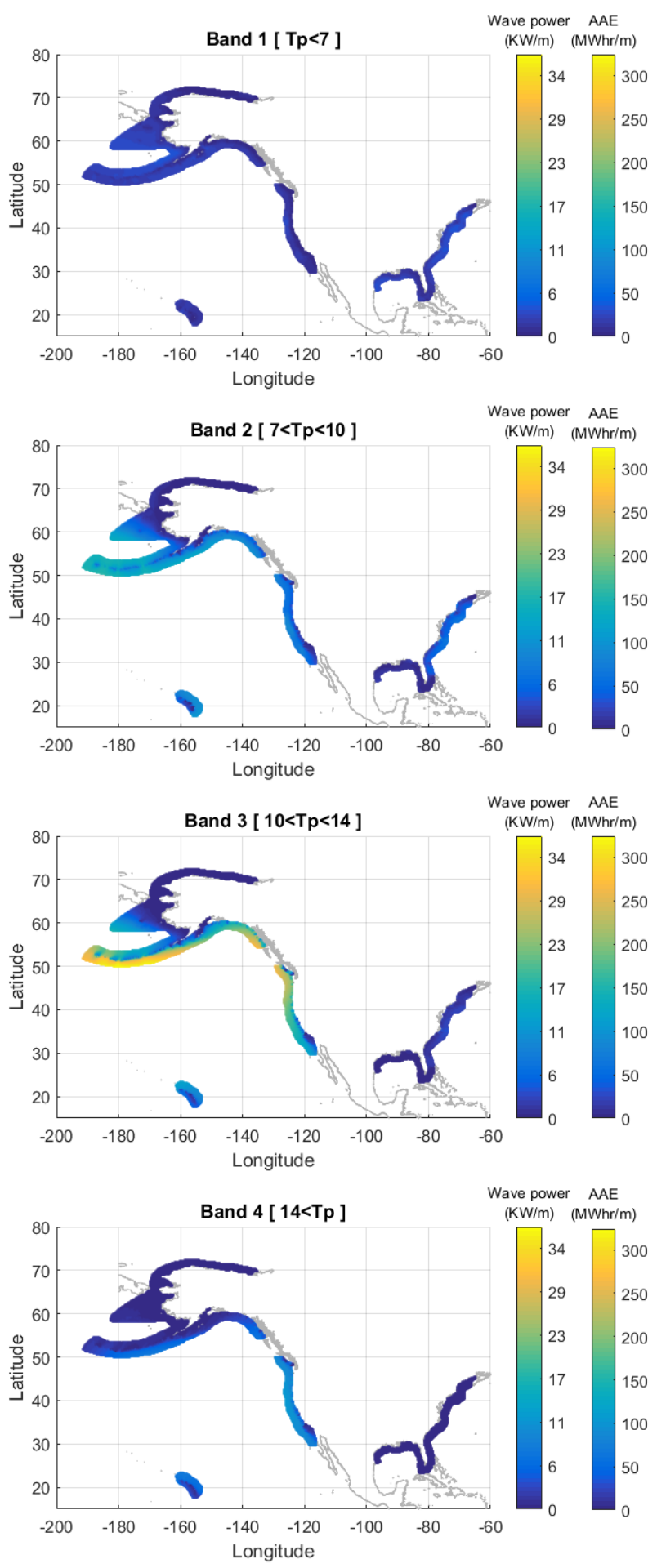

Fig. 3. Geographic distribution of wave energy resource classes 


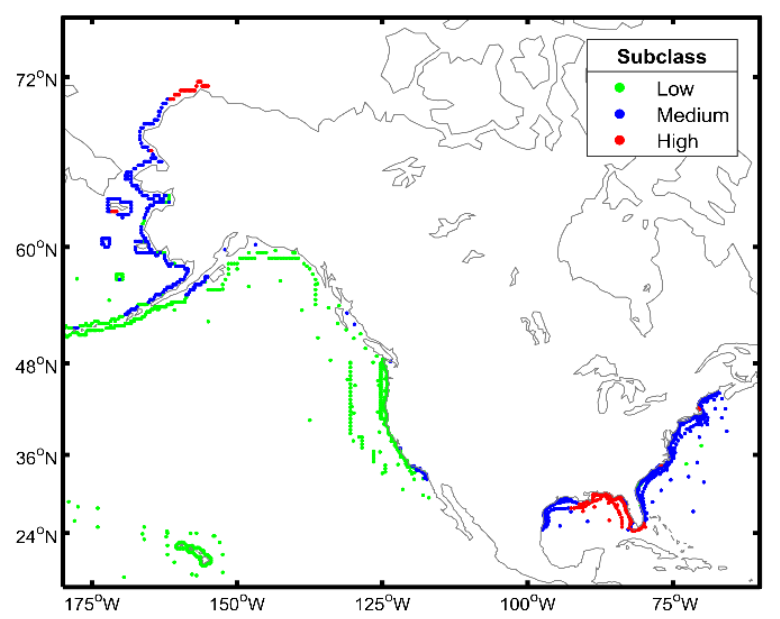

Fig. 4 - Geographic distribution of wave climates based on relative risk classes: low $\left(H_{S_{50}} / H_{s_{\text {mean }}} \leq 5\right)$, medium $\left(5<H_{S_{50}} / H_{s_{\text {mean }}}<8\right)$, high $(8 \leq$ $\left.H_{s_{50}} / H_{\text {smean }}\right)$.

Fig. 5 shows the mean sea states $\left(H_{s}, T_{e}\right)$ for these thirty wave sites relative to all sites for which full spectra outputs are available from NOAA's thirty-year hindcast. It also highlights the variation of average sea states within each climate and resource class for comparison with wave climates in coastal water of the UK and Ireland [2]. The West Coast sites, clustered around $T_{e}=10$ seconds, are mostly high energy sites with $H_{s}$ exceeding $2 \mathrm{~m}$, except for two sites (3 and 6) where $H_{s}$ is just over $1 \mathrm{~m}$. These relatively low energy (Class 2) West Coast sites are located off the coast of southern California. The East Coast sites are clustered around $T_{e}=6$ seconds with $H_{s}$ from about $1 \mathrm{~m}$ to just over $1.5 \mathrm{~m}$, with the exception of site 10 , which is an anomalous site with similar energy characteristics as Gulf Coast sites. Finally, the Gulf Coast sites are clustered around $T_{e}=4$ seconds with $H_{s}$ from about $0.5 \mathrm{~m}$ to $1 \mathrm{~m}$.

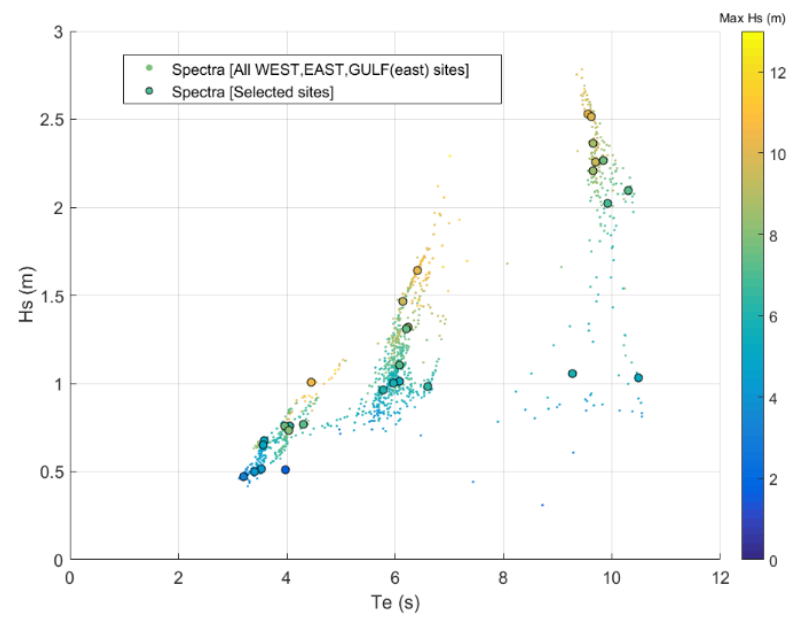

Fig. 5 - Mean sea states for study sites in three wave climates.

\section{WEC PERFORMANCE OPTIMIZATION}

In order to derive a distribution of optimal WEC designs for different wave climates (resource classes), a design optimization algorithm was applied to the Reference Model 3 (RM3) WEC with modelled wave spectra as inputs from the aforementioned thirty sites. The RM3 is a two-body point absorber, which was originally designed for a site off the shore of Eureka, California [4]. The scaling factor, $\lambda$, is a length scale with $\lambda=1$ corresponding to a RM3 point absorber with a $20 \mathrm{~m}$ diameter float, and a spar length of $42 \mathrm{~m}$.

The procedure for deriving the distribution of optimal scaling factors for each wave climate is illustrated in Fig. 6. First, a set of frequency response functions (FRFs) are derived from the boundary element tool WAMIT [5] for the nominal scale of the RM3 WEC $(\lambda=1)$. The RM3 scale $(\lambda)$ is then optimized for thirty-years of modelled 3-hourly wave spectra inputs for each wave climate to maximize the cost parameter.

$$
\lambda=\operatorname{argmax}\left(\frac{E}{1+\lambda^{3}}\right)
$$

using the optimization algorithm (fminbnd in MATLAB [4]), where $E$ is the energy converted (absorbed) in the sea state, and the denominator, $1+\lambda^{3}$, is a proxy for cost.

This procedure considers two optimization cases: one using optimal resistive damping control (RDC), where the PTO input is a simple constant proportion of velocity; and the other using complex conjugate control (CCC), applying a pseudospectral approach to find the optimal impedance matching control [7]. These two approaches return different powers for a given device scale and sea state. The optimal device scale is dependent on the control applied [8], with the RDC optimization delineating the lower bound for energy absorption, and CCC optimization its upper bound [9].

Fig. 7 shows the optimal scale for RM3 devices at each of the ten selected sites within the West, Gulf and East Coast climates. The median device scale is shown along with variance bars indicating variations in the optimal design scaling factor due to hourly, seasonal and inter-annual variations in the wave spectra. As expected, the largest scaling parameters are observed for the West Coast sites, followed by those observed for the East and Gulf Coasts.

Median scaling factors are found to be remarkably similar between most sites within each region, likely due to similar energy distributions within the partitioned period bands, and partly reflected in the similar mean $T_{e}$ values among the ten sites within each wave climate. This explanation appears to be especially justified given the two Class 2, West Coast, "outlier" sites, 3 and 6, have similar scaling factors compared to the other Class 3 sites in this wave climate because of similar energy distributions within partitioned period bands. In other words, the optimal design scaling factor is not as sensitive to the amount of available energy. Explaining the result for the East Coast site 10, however, is difficult as this site exhibits a similar median design scale factor to the other East Coast sites despite exhibiting wave climate characteristics as the Gulf Coast; specifically, the same characteristic energy distribution. More investigation is needed to explain these results, including the possibility of an error in the procedure. 


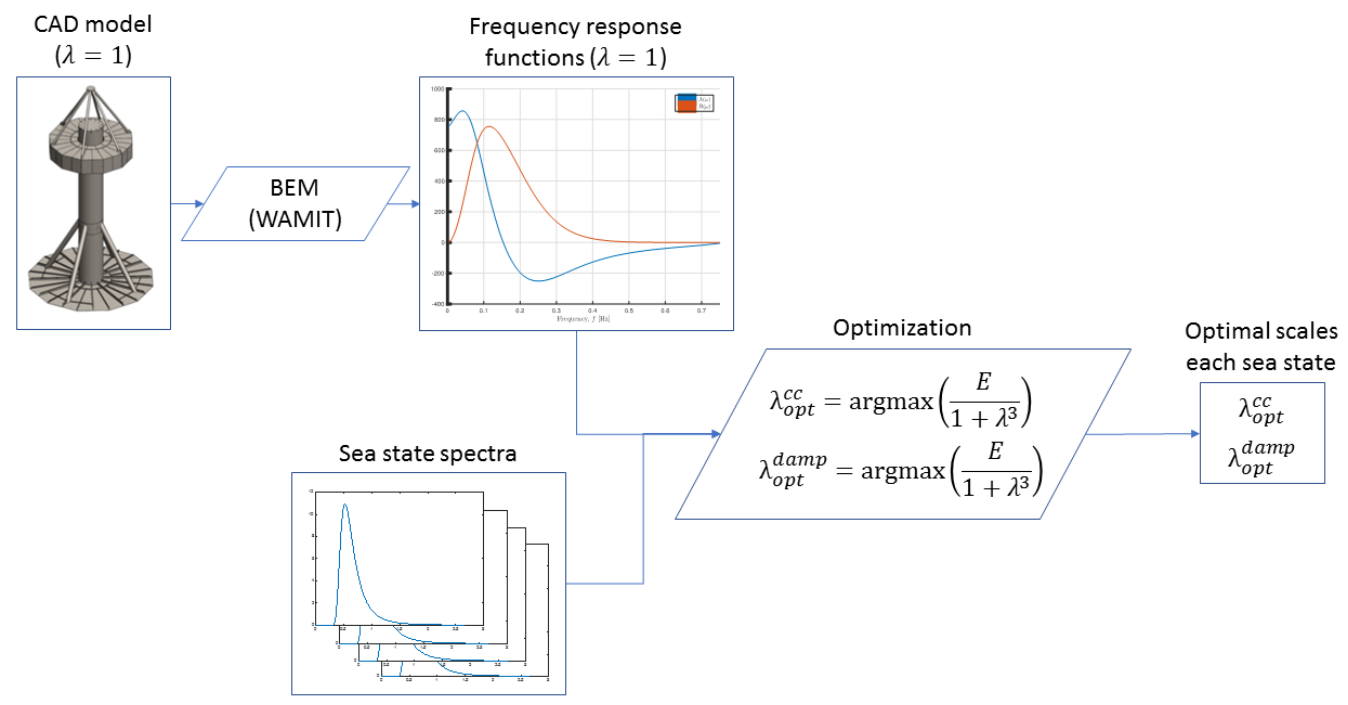

Fig. 6 - RM3 WEC scale optimization process.

The optimal scaling factors are not sensitive to the control approach for the West Coast sites, with the exception of site 1. However, for the East and Gulf Coast sites, optimization with CCC generally provides significant benefits by reducing WEC design scales using optimal damping. For the Gulf Coast sites, it also reduces the variability in median optimal scaling factor among the ten sites.

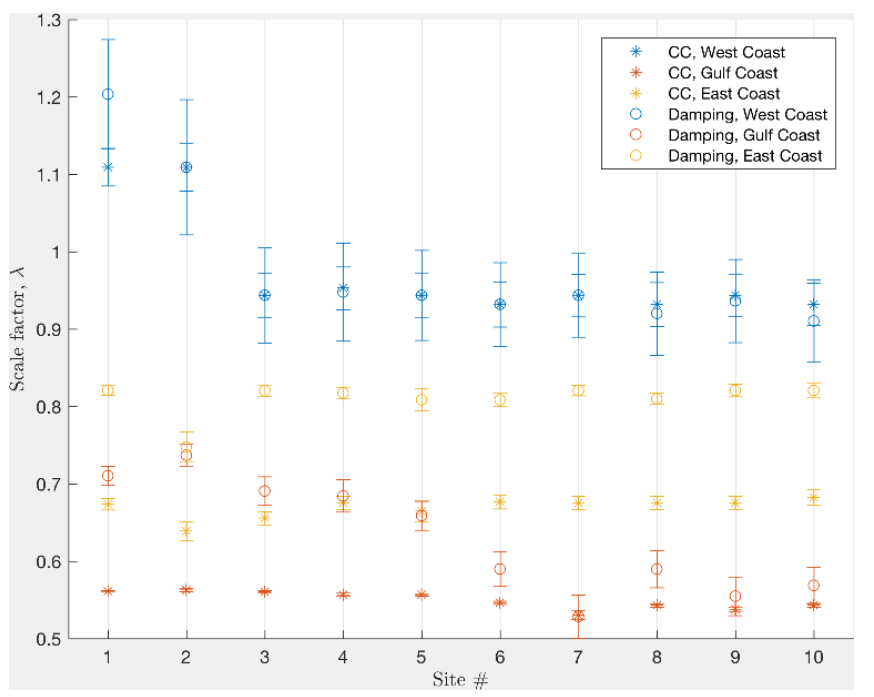

Fig. 7 - Optimal RM3 scale $(\boldsymbol{\lambda})$ for sites in West, Gulf and East Coast regions. Error bars show seasonal variation of site. Note that sites do not correspond between regions.

The distributions of derived optimal WEC designs are also examined by plotting histograms that include scaling factors for approximately thirty thousand sea states within each of the three wave climates. These frequency histograms are shown in Fig. 8, for CCC optimization, and Fig. 9, for RDC optimization. In both plots the distributions for each wave climate overlap, but the population of optimal scales for each climate clearly shift from high values for the West Coast, where the energy is concentrated in moderate swells (Band 3), to the Gulf Coast, where the energy is concentrated in local wind seas (Band 1). The shapes of these frequency histograms are particularly useful as a measure of the feasibility of WEC classification. Unimodal distributions, near-normal distributions in particular, indicate success because variations can be explained by inherent hourly and seasonal variations in sea states. These plots show the effect of the optimization control approach on the distribution shape.

For CCC optimization, three distinct distribution shapes are observed for each region: a bi-modal distribution for the West Coast, a right-skewed distribution for the East Coast, and a leftskewed distribution for the Gulf Coast. The bi-modal distribution for the West Coast suggests the need for two different standard design classes, but this result needs to be investigated further because it does not appear to be supported by the results in Fig. 7, for which the only outliers, sites 1 and 2, are typical Class 3 West Coast sites.

For optimization with optimal RDC, the West Coast distribution shape remains predominantly bi-modal, but the East Coast distribution changes to a left-skewed shape, and the left-skewed Gulf Coast distribution becomes a "dog food" distribution; which would indicate that a standard design class for this climate is not possible.

Although preliminary, these results show that the derived distributions for optimal scales are generally unique for distinct wave climates (resource classes). While the shapes of these distributions are not normal, they are generally monotonic and only slightly skewed. As one would expect, the optimal scaling factor (size) of the WEC appears to be driven mainly by the dominant period band for each wave climate (resource class). For the West Coast, moderate period swell contributes most of the energy in Band 3, $\left(10 \mathrm{~s}<T_{p}<14 \mathrm{~s}\right)$, even for the two Class 2 sites, 3 and 6. For the East Coast most of the energy is in the short period swell in Band 2, (7s $\left.<T_{p}<10 \mathrm{~s}\right)$, and for the Gulf Coast, low period local wind seas in Band $\left(T_{p}<7 \mathrm{~s}\right)$. 


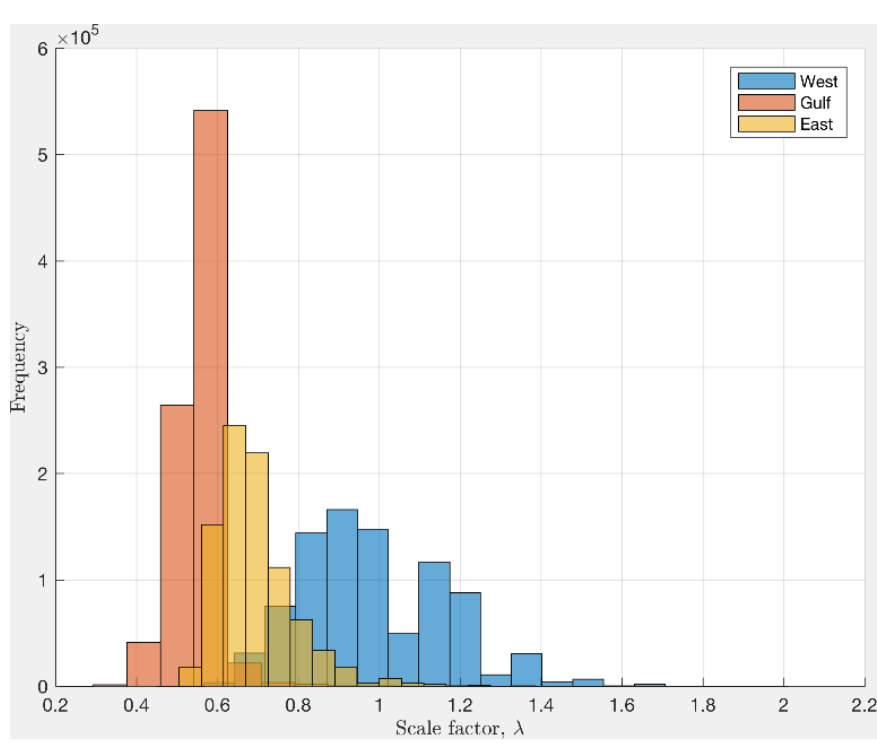

Fig. 8 - Histogram of optimal scales with complex-conjugate control for all sea states analyzed on West, Gulf and East Coast regions.

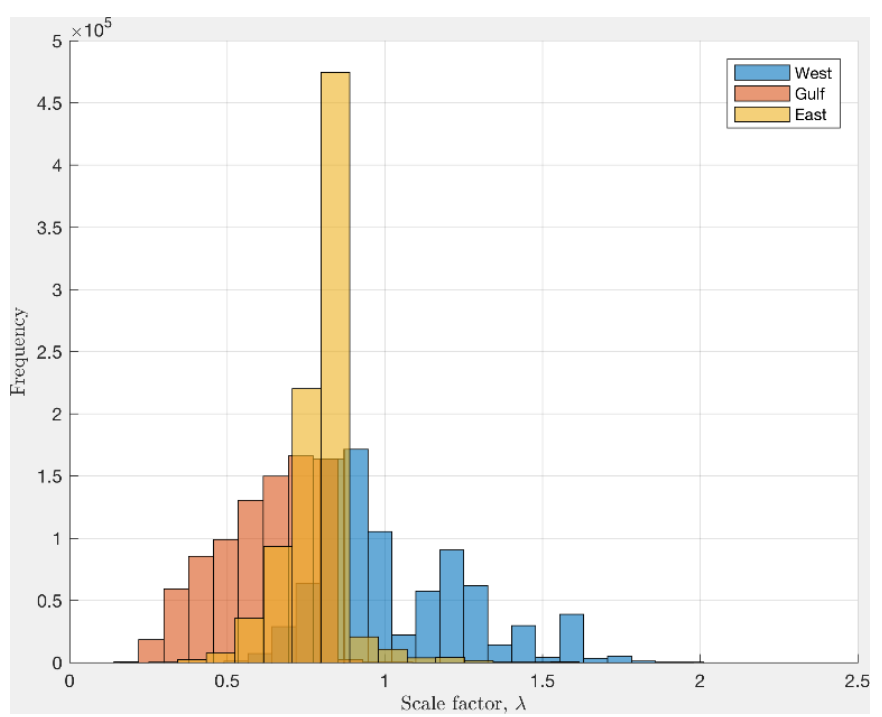

Fig. 9 - Histogram of optimal scales with resistive damping control for all sea states analyzed on West, Gulf and East Coast regions.

\section{WEC DESIGN LOADS}

In order to investigate the variation (spread) of extreme load responses within a resource class, representative design load estimates for the nominal scale of the RM3 WEC $(\lambda=1)$ were derived for three NDBC buoy sites in the West Coast climate. To characterise both the sites of interest and representative design load metrics, the WEC Design Response Toolbox (WDRT) [10] was used. For the former, the WDRT includes a routine for creating environmental contours of extreme sea states based on the principle components analysis (PCA) methodology [11], allowing the characterization of the joint probability distribution of sea state variables of interest. The resulting samples can be used for numerical or physical model simulations analysing the design response of WECs characterized through complementary cumulative distribution functions [12]. Fig. 10 shows the resulting characterization for three National Data Buoy Center (NDBC) locations in the West Coast region, NDBC station 46011 (Southern California), 46022 (Northern California), and 46041 (Oregon). The locations were selected to provide an indication of the interregion variation that may be expected, and correspond approximately to the West Coast sites 7, 8 and 9 in the Section III analysis.
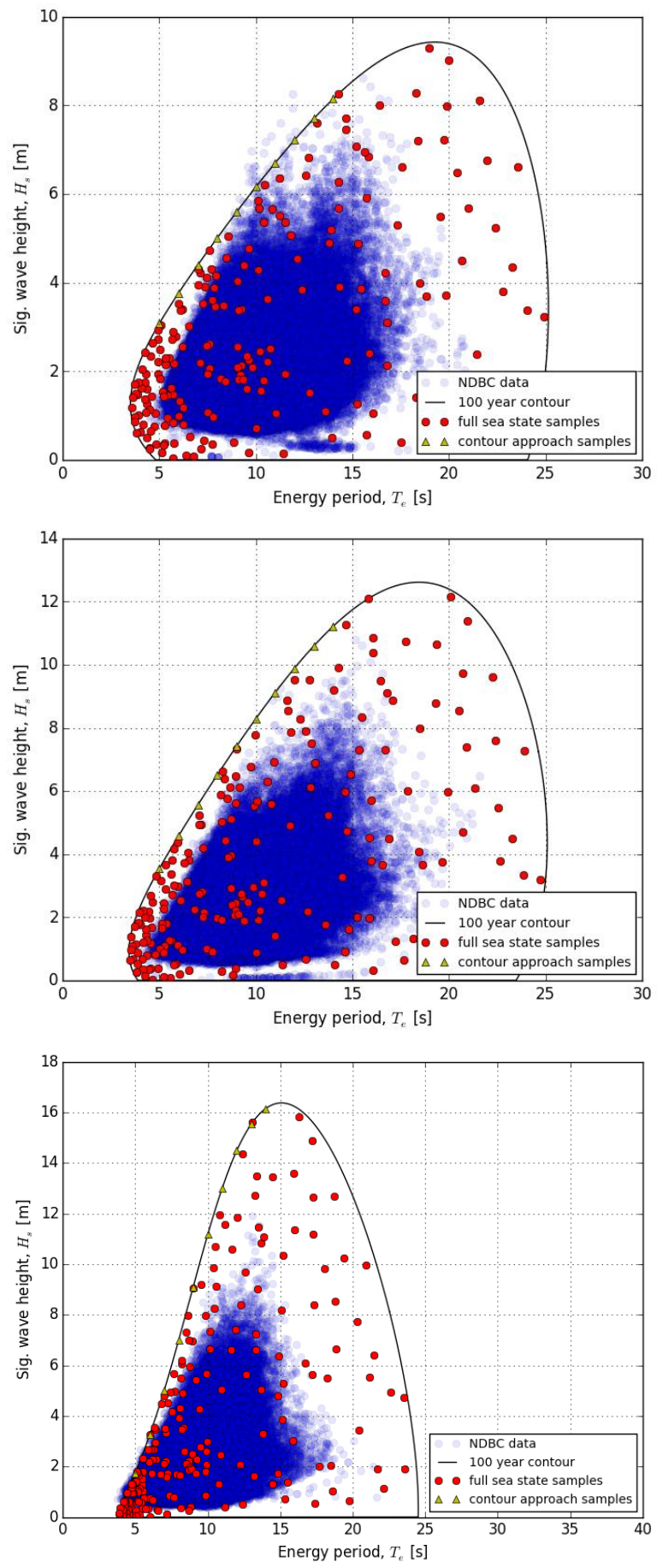

Fig. 10 - Environmental characterization of the NDBC sites: 46011 (top), 46022 (middle) and 46041 (bottom). 
For each site, 200 samples (see red dots in Fig. 10) were selected using the PCA approach to characterize the 100-year return environmental conditions. For each of the 200 sea state samples (per site), 3-hour loading estimates were obtained from a WEC-Sim linear model of the nominal scale RM3 WEC. The corresponding 200 short-term extreme response distributions (estimated from Weibull tail fits) were obtained for key design variables, namely the wave excitation force (surge and heave) acting the prime mover (float body) and the power take-off (PTO) force. The formulation of a Weibull tail fit short-term extreme distribution CDF is given in Equation (5), where $x$ is the variable of interest, $s c$ is the scale parameter, $s h$ is the shape parameter and $N$ is the number of peaks counted in the time-series. It is noted that $s c, s h$, and $N$ depend on the shortterm response sample.

$$
C D F_{\text {Weibull-tf }}=\left(1-e^{-\left(\frac{x}{s c}\right)^{s h}}\right)^{N}
$$

Having derived the 200 device short-term extreme response probabilities for each site, site-specific long-term extreme response distribution were estimated by a weighted-average of the complementary cumulative distribution function (CCDF), as described in Equation (6), where $w_{i}$ is the weight given to each short-term sample $\left(\sum_{i=1}^{i=200} w_{i}=1\right)$.

$$
C C D F_{\text {long-term }}=\sum_{i=1}^{i=200} w_{i}\left(1-C D F_{\text {Weibull-tf }, i}\right)
$$

The long-term CCDF is sometimes referred to as a 'survival function'. Examples of the resulting survival functions are illustrated in Fig. 11. These plots also show the individual short-term survival response functions.

There is a significant variation across the site both in terms of annual average wave power density $(46011-25.6 \mathrm{~kW} / \mathrm{m}$; $46022-36.8 \mathrm{~kW} / \mathrm{m} ; 46041-31.8 \mathrm{~kW} / \mathrm{m})$ and in terms of extreme loads (see Fig. 11). However, there are no clear correlations between the dimensional load metrics (as for the float surge excitation force presented in Fig. 11) and the annual wave power. This problem was addressed by nondimensionalizing the design loads obtained as a function of the target return period using a linear incident wave force (FroudeKrylov component), given by $\rho g A_{c}|\eta|_{r}$, where $A_{c}$ is the characteristic cross-sectional area and $|\eta|_{r}$ the long-term wave elevation for return period $r$.

Fig. 12 to Fig. 14 show representative results, including the dimensional wave elevation and float heave exicting force metrics, the non-dimensional design loads for the float (heave and surge), and the non-dimensional PTO design load, respectively. The largest difference between the nondimensional design loads (approximately 7\% variation for the 100 -year return non-dimensional float heave exciting force) is substantially lower than the inter-regional wave power variation across the three sites, which suggests that the longterm linear incident wave force for return period $r$ can be used for classification purposes.
This preliminary result indicates the suitability of the classification system to provide guideline estimates of key design load metrics as a function of the design variable, WEC design and site characteristics. Future work may address the combined effects of WEC and site scaling, as well as the influence of nonlinearities in the overall results.
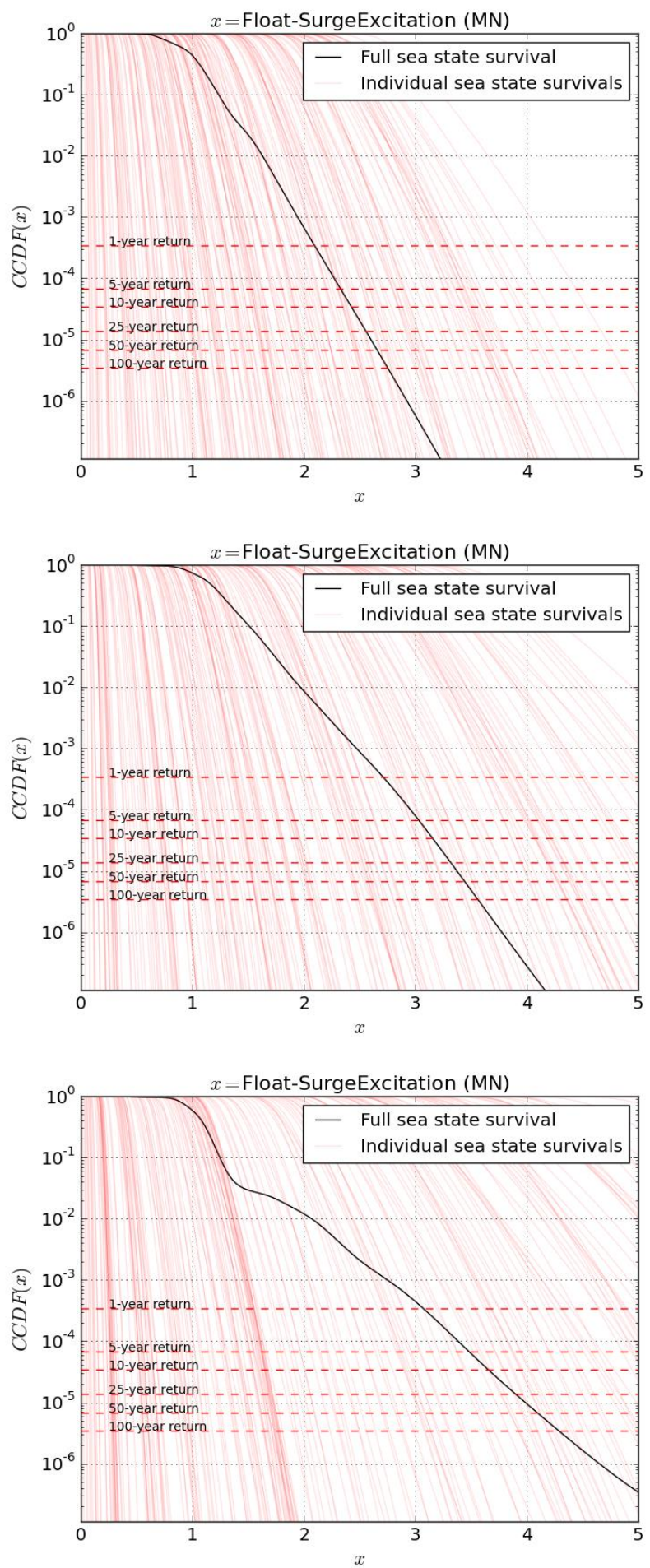

Fig. 11 - Examples of the resulting survival functions (float surge exciting force) for multiple NDBC sites: 46011 (top), 46022 (middle) and 46041 (bottom). 

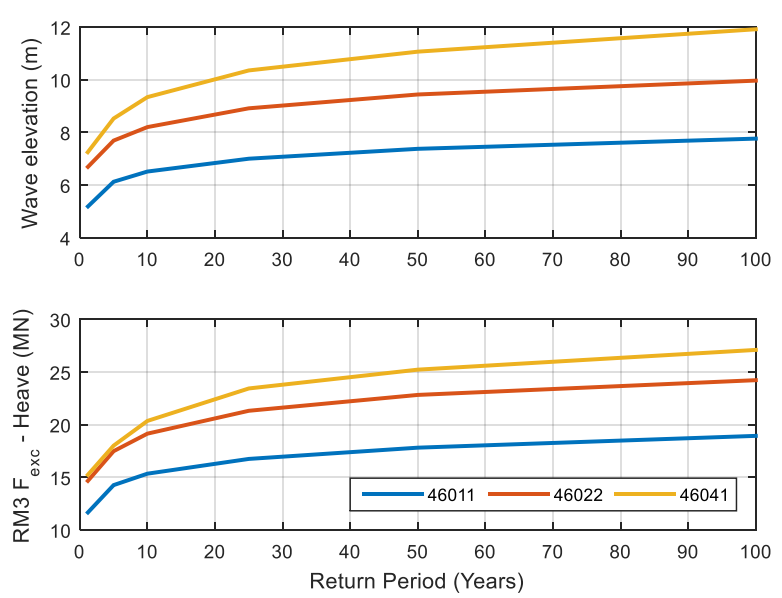

Fig. 12 - Long-term return values of the wave elevation (top) and RM3 float heave exicting force (bottom) for 3 NDBC sites (West Coast).
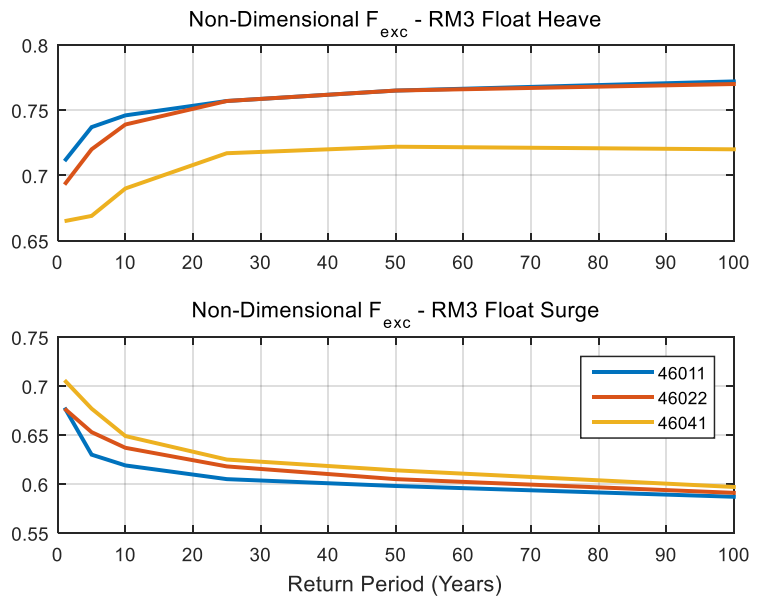

Fig. 13 - Non-dimensional long-term return values of the RM3 float heave (top) and surge (bottom) exicting force for 3 NDBC sites.

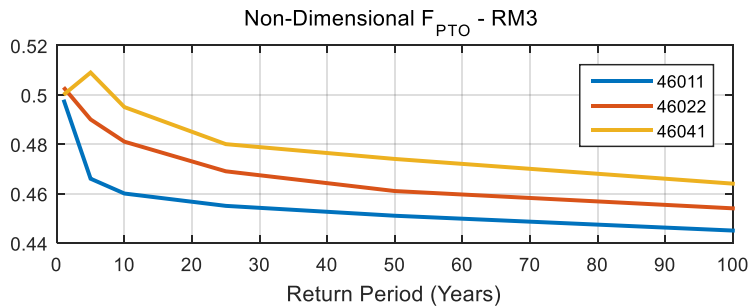

Fig. 14 - Non-dimensional long-term return values of the RM3 PTO force for 3 NDBC sites.

\section{CONCLUSIONS}

In the present study, a classification system for U.S. wave resources reveals unique regional wave climate characteristics and the potential to develop standard WEC designs (a WEC classification system). The feasibility of WEC classification is examined through: 1) Evaluation of derived distributions of optimized WEC design scaling factors for an RM3 point absorber operating in three different wave climates spanning the full range of resource class conditions; and 2) Evaluation of WEC extreme load responses for three Class 3 wave sites located in the West Coast wave climate.
Preliminary results indicate that WEC classification is a potentially viable design strategy, with standard design classes serving broad regional wave climates because wave energy contributions are generally concentrated within distinct partitioned period bands for these climates. Optimal WEC designs appear to be driven mainly by the dominant period bands observed for three U.S. wave energy resource classes, high energy Class 3 sites along the West Coast with most of the energy contribution in moderate period swell, moderate energy Class 2 sites along the East Coast with most of the energy contribution in short period swell, and low energy sites along the Gulf Coast with most of the energy contribution in low period local wind seas. The WEC design classes for these three wave climates reflect these dominant period bands. Based on these preliminary results for an RM3 point absorber optimizing the performance function prescribed with $\mathrm{CCC}$, the following standard design classes (by float diameter) would generally apply: An $18 \mathrm{~m}$ diameter $(\lambda=0.9)$ WEC for the West Coast, a $14 \mathrm{~m}$ diameter $(\lambda=0.7)$ WEC for the East Coast, and a $11 \mathrm{~m}$ diameter $(\lambda=0.55)$ WEC for the Gulf Coast.

The WEC response to extreme loads was found to vary considerably within the most energetic of the resource classes examined in the West Coast climate, suggesting the need for these standard design classes to meet structural design requirements based on the upper limits of load response within a given resource class. However, the observed load metric variation is lower than the inter-region resource variations, which provides a preliminary indication of the suitability of the classification system to provide guideline estimates of key design load metrics as a function of the target design variable, WEC design and site characteristics.

This initial investigation of standard WEC design feasibility is encouraging, but additional work is needed to verify observed trends, their underlying mechanisms, and unexplained anomalies. Future work, including recommendations by [2], may include:

- Use of summary statistics for derived distributions as measures of classification performance.

- Extension of the analyses for WEC design optimization and extreme load response using a larger population of hindcast wave spectra from more sites within each wave climate and resource class.

- Evaluation of derived distributions of WEC extreme load responses, as was done for scaling factors.

- Refinement of the performance function, and an investigation of its effects on the derived optimized scaling factor distributions.

\section{ACKNOWLEDGMENTS}

Sandia National Laboratories is a multi-mission laboratory managed and operated by National Technology and Engineering Solutions of Sandia, LLC., a wholly owned subsidiary of Honeywell International, Inc., for the U.S. Department of Energy's National Nuclear Security Administration under contract DE-NA0003525. 


\section{REFERENCES}

[1] IEC. International Standard: Wind turbines - Part 1: Design Requirements. IEC 61400-1:2500(E). Edition 3.1, April 15, 2014.

[2] Cruz J. WEC Classes: Preliminary Studies, Proceedings of the 11th European Wave and Tidal Energy Conference 6-11th Sept 2015, Nantes, France.

[3] Chawla, A., Spindler, D. M., \& Tolman, H. L. , 2013, "Validation of a thirty year wave hindcast using the Climate Forecast System Reanalysis winds," Ocean Modelling, Vol. 70, pp. 189-206.

[4] V. S. Neary et al., "Methodology for Design and Economic Analysis of Marine Energy Conversion (MEC) Technologies," Sandia National Laboratories Report SAND2014-9040, March 2014.

[5] WAMIT, WAMIT User Manual, 7th ed., Chestnut Hill, MA, 2012. [Online]. Available: http://www.wamit.com/manual.htm

[6] MATLAB, 2016. 9.0.0.34136 (R2016a). The MathWorks Inc., Natick, Massachusetts.

[7] Bacelli, Giorgio, and John V. Ringwood. "Numerical optimal control of wave energy converters." IEEE Transactions on Sustainable Energy 6.2 (2015): 294-302.

[8] Garcia-Rosa, P. B., and Ringwood, J. V., 2016. "On the sensitivity of optimal wave energy device geometry to the energy maximizing control system". IEEE Transactions on Sustainable Energy, 7(1), Jan, pp. 419426.

[9] Wilson, D. G., Bacelli, G., Coe, R. G., Bull, D. L., Abdelkhalik, O., Korde, U., and Robinett, R., 2016. A comparison of WEC control strategies. Tech. Rep. SAND2016- 4293, Sandia National Laboratories, Albuquerque, NM, May

[10] R.G. Coe, C. Michelen, A. Eckert-Gallup, Y. Yu and J. van Rij, "WDRT: A toolbox for design-response analysis of wave energy converters," Proceedings of the 4th Marine Energy Technology Symposium (METS), Washington, DC, 2016

[11] Eckert-Gallup, A., C. Sallaberry, A.R. Dallman and V.S. Neary (2016) Modified inverse first-order reliability method (I-FORM) for predicting extreme sea states. Ocean Engineering112 (2016) 307-319. http://dx.doi.org/10.1016/j.oceaneng.2015.12.018

[12] Michelen, C. and R. Coe, Comparison of methods for estimating shortterm extreme response of wave energy converters, in: OCEANS $2015-$ MTS/IEEE Washington, IEEE, Washington, D.C., 2015. 
